\title{
Trinucleotide repeat length and progression of illness in Huntington's disease
}

Karl Kieburtz, Marcy MacDonald, Charles Shih, Andrew Feigin, Kim Steinberg, Kathy Bordwell, Carol Zimmerman, Jayalakshmi Srinidhi, Jenny Sotack, James Gusella, Ira Shoulson

\begin{abstract}
The genetic defect causing Huntington's disease (HD) has been identified as an unstable expansion of a trinucleotide (CAG) repeat sequence within the coding region of the IT15 gene on chromosome 4. In 50 patients with manifest $H D$ who were evaluated prospectively and uniformly, we examined the relationship between the extent of the DNA expansion and the rate of illness progression. Although the length of CAG repeats showed a strong inverse correlation with the age at onset of $H D$, there was no such relationship between the number of CAG repeats and the rate of clinical decline. These findings suggest that the CAG repeat length may influence or trigger the onset of HD, but other genetic, neurobiological, or environmental factors contribute to the progression of illness and the underlying pace of neuronal degeneration.
\end{abstract}

( $($ Med Genet 1994;31:872-874)

An unstable expansion of trinucleotide (CAG) repeats in the IT 15 gene has been identified as the genetic defect underlying the inheritance of Huntington's disease (HD). ${ }^{1}$ While the number of CAG repeats in IT15 ranges normally from nine to 37 , the number of repeats in affected HD persons ranges from 37 to $102 .^{2-7}$ A strong inverse correlation between the number of trinucleotide repeats and the age at onset of HD has been shown; however, no apparent relationship has been found between the number of repeats and clinical manifestations of illness. ${ }^{48}$

Early age at onset of HD has been considered a harbinger of more rapid progression of illness, but a recent analysis of 1000 patients with HD failed to confirm such a relationship. ${ }^{9}$ The HD Functional Capacity Scale (HDFCS), as reflected by its aggregate score of total functional capacity (TFC), has been found to be a valid and reliable measure of the severity of HD. ${ }^{1011}$ Using TFC as an index of illness progression, we similarly found no correlation between the age at onset of HD and the rate of illness progression. ${ }^{12}$

A reliable biological marker or predictor of the rate of illness progression would be useful for evaluating potential therapies for $\mathrm{HD}$ as well as for caring for patients and families. The extent of the underlying genetic defect, as measured by the number of CAG repeats, is a potential marker of illness progression. We therefore examined the relationship between the number of CAG trinucleotide repeats in the IT15 gene and the onset and prospective course of HD.

\section{Methods}

We studied patients with manifest HD to determine the rate of illness progression and attendant clinical features. A single examiner (IS) prospectively evaluated each patient using the HDFCS scale. The total functional capacity (TFC) score, ranging from 13 (normal) to 0 (severely incapacitated) units, assesses a patient's capacity in relevant functional domains including employability, financial tasks, domestic capacities, and self-care skills. Patients were evaluated on at least two occasions separated by at least six months in the setting of continuing clinical care, while participating in a controlled clinical trial, ${ }^{13}$ or both. All clinical assessments were made independent of any information regarding DNA analysis and CAG repeat length.

Prospectively derived information, including family history, demographic data, and medications, was also obtained from all patients. Age of onset was determined by a single examiner (IS) on the basis of the first reported onset of motor features. After obtaining consent from the patient or guardian, blood samples were obtained from $54 \mathrm{HD}$ patients.

DNA was prepared from frozen blood samples as described previously. ${ }^{2}$ The length of the CAG trinucleotide repeat was determined using the polymerase chain reaction (PCR) to amplify either the CAG and polymorphic CCG stretch as originally described ${ }^{1}$ or with oligonucleotide primers 5'ATGAAGGCCTTCGAGTCCCTCAAGTCCTTC $3^{\prime}$ and 5'GGCGGTGGCGGCTGTTGCTGCTGCTGCTGC3' to amplify just the CAG stretch, as described by Warner et al. ${ }^{14}$ The sizes of the PCR products were determined by comparison with signed products generated in the PCR assay using sequencing cosmid DNAs. The two methods yielded similar results, and only data from the method described by Warner et $a l^{14}$ are presented.

The rate of TFC change for each patient was calculated from the slope of the least squares fitted line, using individual plots of the patient's TFC score over time. Correlations were tested by Pearson coefficients comparing the rate of TFC change, the number of CAG repeats, and the natural log of the age at onset of $\mathrm{HD}$. 


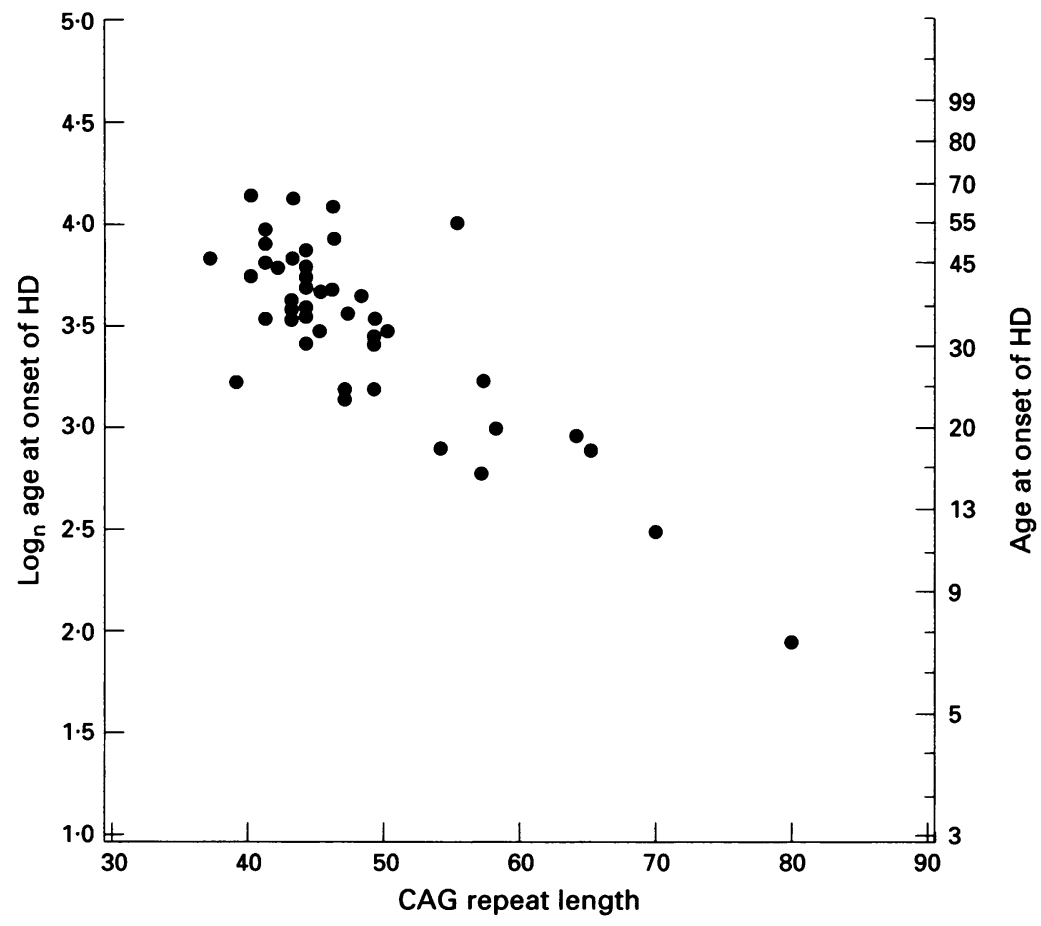

Figure 1 Relationship between age at onset of $H D$ and $C A G$ repeat length.

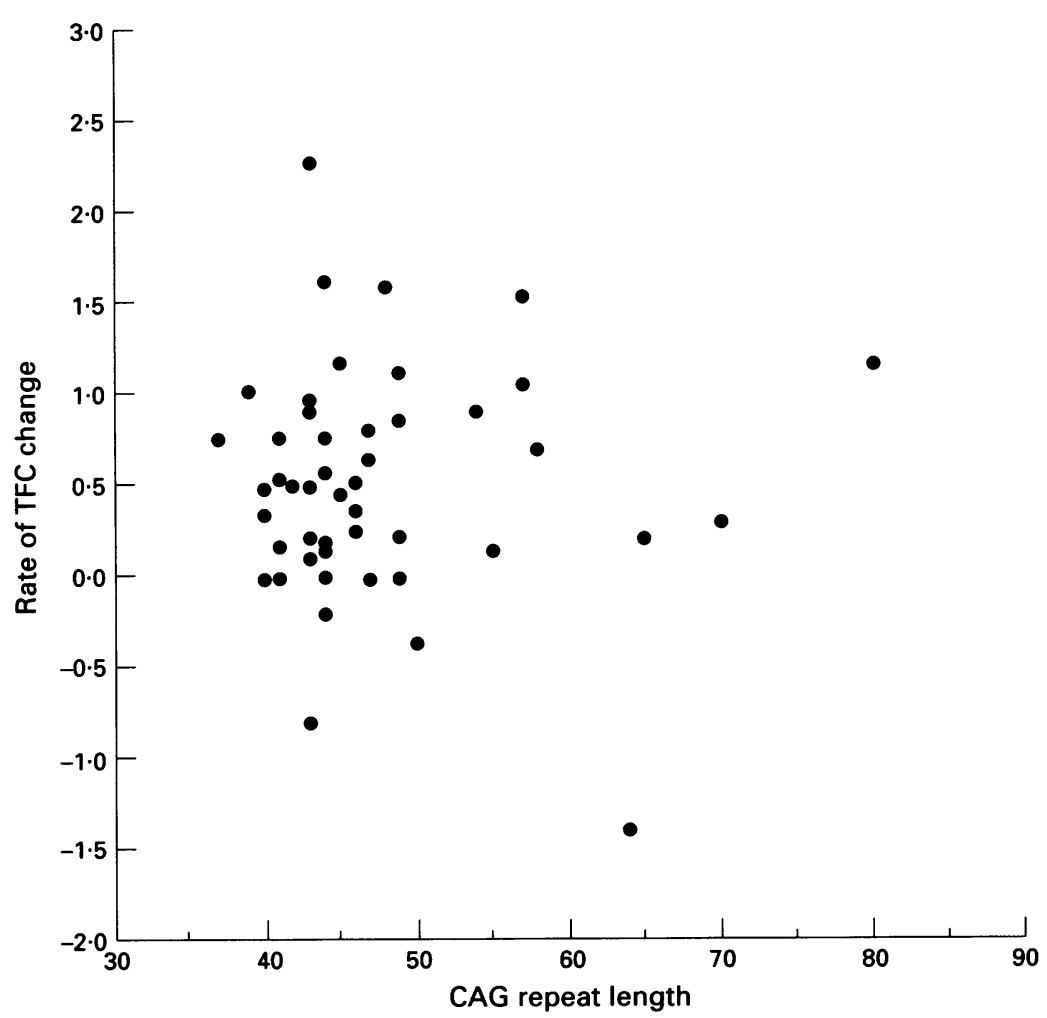

Figure 2 Relationship between annual rate of TFC change (units/year) and CAG repeat length.
16) and were followed for $4 \cdot 4$ (SD 2.9) years (range 0.5 to $13 \cdot 8$ ). The TFC at initial evaluation was $9 \cdot 2$ (SD $2 \cdot 1$ ) (range 2 to 13 ), and the TFC at final evaluation was $6 \cdot 8$ (SD 3.1) (range 0 to 12). The mean rate of TFC decline was 0.5 (SD 0.6) TFC units per year (range $-1 \cdot 4$ to $2 \cdot 3$ ) similar to previous reports of the rate of TFC decline in $\mathrm{HD} .{ }^{10}$ Only four subjects showed any improvement in TFC scores (negative rate of TFC change) during the period of observation.

The average CAG repeat length was 47.5 (SD 8.3) (range 37 to 80). There was a strong inverse correlation between CAG repeat length and age at onset: $r=-0.82, p<0.0001$ (fig 1), similar to that found by other investigators. ${ }^{23}$ In contrast, the relationship between CAG repeat length and the rate of TFC decline (fig 2) was virtually a flat line: $r=0.01, p=0.97$. There was also no relationship between age at onset and the rate of TFC decline (fig 3 ).

\section{Discussion}

In this prospective study, we confirmed the strong relationship between CAG repeat length in the IT15 gene and the age at onset of HD. However, consistent with the report of Roos et $a l,{ }^{9}$ we found no relationship between age at onset of HD and severity of illness. In a previous study we examined the rate of functional decline in a cohort of 129 prospectively evaluated patients with $\mathrm{HD}$ and, contrary to previous reports, ${ }^{15}$ found no correlation between functional decline and age at onset, body weight, gender of affected parent, or neuroleptic use. ${ }^{12}$

Our current study failed to establish any relationship between the number of CAG repeats and the rate of functional decline as assessed by the TFC scale. These findings suggest that the range of HD severity is relatively uniform across a large spectrum of age at onset and CAG repeat length. However, we found considerable variability in the rate of decline in our cohort, ranging from relatively stable illness to declining at greater than two TFC units per year (fig 2). Taken together, these observations suggest that the number of CAG repeats is a strong predictor of the age at onset of HD but not of the pace of disease progression. Thus, the CAG repeat length may play an important role in the initiation of the clinical features of $\mathrm{HD}$, but there appear to be other contributing genetic, neurobiological, or environmental factors that modify and influence the subsequent progression of disease.

The lack of association between the number of CAG repeats and the progression of illness limits the use of CAG repeat length as a reliable predictor of the clinical course. Some of our patients with relatively few repeat lengths experienced a rapid clinical decline while some patients with high repeat lengths had a relatively slow clinical course. These findings have implications for genetic counselling and the appropriate interpretation of genetic test results in patients with symptomatic HD. These observations may extend to asymptomatic persons at risk for $\mathrm{HD}$, but need to be confirmed in such a population. 


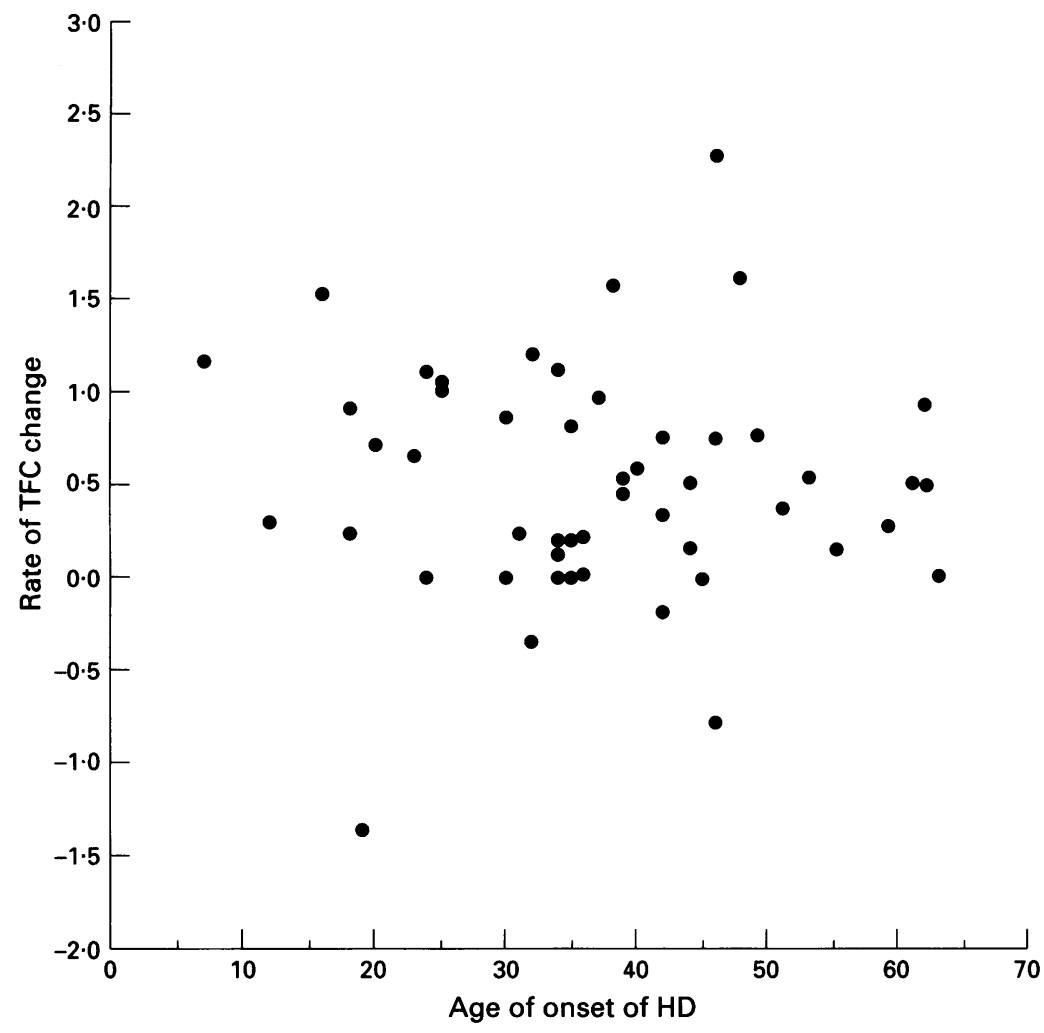

Figure 3 Relationship between annual rate of TFC change and age at onset of HD. This work was supported in part by USPHS grants NS-17978,
NS-16367, the Clinical Research Center of the University of NS-16367, the Clinical Research Center of the University of
Rochester (RR-00044), and the Lucille P Markey Charitable Trust.
1 Huntington's Disease Collaborative Research Group. A novel gene containing a trinucleotide repeat that is expanded and unstable on Huntington's disease chromosomes. Cell 1993;71:971-83.

2 Duyao M, Ambrose C, Myers R, et al. Trinucleotide repeat length instability and age of onset in Huntington's disease. length instability and age

3 Snell RG, MacMillan JC, Cheadle JP, et al. Relationship between trinucleotide repeat expansion and phenotypic between trinucleotide repeat expansion and phenotypic

4 Andrew SE, Goldberg YP, Kremer B, et al. The relationship between trinucleotide (CAG) repeat length and clinical between trinucleotide (CAG) repeat length and clinical
features of Huntington's disease. Nature 1993;4:398-403.

5 Stine OC, Pleasant N, Franz ML, Abbott MH, Foistein SE, Ross CA. Correlation between the onset age of Huntington's disease and length of the trinucleotide repeat in IT-15. Hum Molec Genet 1993;2:1547-9.

6 Craufurd D, Dodge A. Mutation size and age at onset in Huntington's disease. F Med Genet 1993;30:1008-11.

7 Simpson SA, Davidson MJ, Barron LH. Huntington's disease in Grampian region: correlation of the CAG repeat number and the age of onset of the disease. $\mathcal{F}$ Med Genet 1993;30:1014-17.

8 MacMillan JC, Snell RG, Tyler A, et al. Molecular analysis and clinical correlations of the Huntington's disease mutation. Lancet 1993;342:954-8.

9 Roos RAC, Hermans J, Vegter-van der Vlis $M$, van Ommen GJB, Bruyn GW. Duration of illness in Huntington's disease is not related to age at onset. $\mathcal{F}$ Neurol Neurosurg disease is not related to age at

10 Young AB, Shoulson I, Penney JB, et al. Huntington's disease in Venezuela: neurologic features and functional disease in Venezuela: neurologic

11 Shoulson I, Kurlan R, Rubin AJ, et al. Assessment of functional capacity in neurodegenerative movement disorders: Huntington's disease as a prototype. In: Munsat TL, ed. Quantification of neurologic deficit. Boston: Butterworths, 1989:285-309.

12 Feigin A, Kieburtz K, Como P, et al. Predictors of disease progression in Huntington's disease (HD). Proceedings of the 15th International World Federation of Neurology Workshop on Huntington's Disease, 2 September 1993:A53.

13 Shoulson I, Odoroff C, Oakes D, et al. A controlled clinical trial of baclofen as protective therapy in early Huntington's disease. Ann Neurol 1989;25:252-9.

14 Warner JP, Barron JH, Brock DJH. A new polymerase chain reaction (PCR) assay for the trinucleotide repeat that is unstable and expanded on Huntington's disease chromosomes. Mol Cell Probes 1993;7:235-9.

15 Myers RH, Sax DS, Koroshetz WJ, et al. Factors associated with slow progression in Huntington's disease. Arch Neurol 1991;48:800-4. 